\title{
Microfluidic Chips Etching Process on Soda-lime Glass
}

\author{
Guanglong Wang \\ Department of Mechanical Engineering \\ College \\ Shijiazhuang 050003, China \\ glwang2005@163.com
}

\author{
Jianhui Chen \\ Department of Mechanical Engineering \\ College \\ Shijiazhuang 050003, China
}

\author{
Qichang Li \\ Department of Mechanical Engineering \\ College \\ Shijiazhuang 050003, China
}

Fengqi Gao

Department of Mechanical Engineering College

Shijiazhuang 050003, China

\author{
Yu Dong \\ Department of Mechanical Engineering \\ College \\ Shijiazhuang 050003, China
}

\author{
Shanshan Zhang \\ Institute of Measurement and Testing \\ Technology Shanghai 200230, China
}

\begin{abstract}
The process to manufacture microfluidic chip using quartz glass or Pyrex 7740 glass is expensive and complex. In order to overcome these, save production costs, simplify the process, and shorten the production cycle, soda-lime glass was designed as substrate carrier; positive photoresist $A Z 4620$ was designed as mask layer, to make the microfluidic chip production process efficient, rapid, and low-cost. Some key technique, such as thickness of photoresist, various stages of baking parameters, exposure and developer volume, etching environment and the glass buffered oxide etching (BOE) ratio had been solved. The adhesion of the photoresist with the glass, and photoresist's tolerance time in BOE had been solved as well. With the optimized process, the etching depth can achieve $80 \mu \mathrm{m}$, the minimum feature size could be less than $50 \mu \mathrm{m}$, the sidewall steepness is less than $100^{\circ}$, the flatness error is less than $\pm 1.5 \mu \mathrm{m}$, and the production cycle is only 4 hour.
\end{abstract}

\section{Keywords-microfluidic chips; soda-lime glass; etching}

\section{INTRODUCTION}

In recent years, a microfabrication process: UV thick photoresist lithography has become a popular research area of micro-systems produce [1-4].Silicon wafers, glass and organic polymers are usually selected as the matrix material of microfluidic chips. Glass-based microfluidic chip has been extensive attention in the last ten years [5]. Most studies based on quartz glass and Pyrex 7740 glass today, because of the excellent optical and chemical properties. But they are expensive; the complex process usually needs a masking layer, such as metal layers, polysilicon layer or silicon nitride layer [6]; and the etching rate is slow relatively, in order to

Authors thank to Institute of Nanotechnology and Microsystems for providing technical support. achieve sufficient depth, a long time is needed [7].

To overcome the above problems, this paper proposes a cheap, simple, rapid method of making glassbased microfluidic chip, it's better for mass production. We used ordinary microscopic slides as the substrate, photoresist AZ 4620 as the mask layer. To increase the adhesion of the photoresist with the glass matrix and extend the tolerance time of photoresist mask layer in BOE, and get enough etched depth of microfluidic chip, we fine controlled the glass substrate surface cleaning pretreatment, the photoresist thickness, the various stages of baking time and temperature; to maximize the etch rate, shorten the process cycle, we controlled the $\mathrm{BOE}$ dilution and etching environment; to reduce the influence of microfluidic chips' morphology and improve the surface flatness, we incorporated hydrochloric acid to dissolve the precipitate particles in the corrosion process.

\section{EXPERIMENTAL METHOD}

In this article, the matrix material of microfluidic chips is the commercial available microscopic slide. It's soda-lime glass with complicated ingredient. In general, the chemical formula is expressed as $\mathrm{Na}_{2} \mathrm{O} \cdot \mathrm{CaO} \cdot 6 \mathrm{SiO}_{2}$. The presence of impurities greatly accelerate the etching rate.The etching mask layer is positive photoresist AZ 4620, developer is AZ $400 \mathrm{~K}$ (1:3). All chemical reagents we used were analytical grade. To ensure that the tolerance time in the etching solution and the graphic flatness of the mask layer, we choose BOE (M (HF): M $\left.\left(\mathrm{NH}_{4} \mathrm{~F}\right): \mathrm{M}\left(\mathrm{H}_{2} \mathrm{O}\right)=3: 6: 9\right)$.

We used the thick photoresist wet etching process to complete the glass-based microfluidic chip. Etching 
process is simple, rapid and inexpensive. The process has only four steps as shown in Fig. 1. Step 1, complete the cleaning and drying of the glass substrate then spin coating; Step 2, exposure and development should be finished; Step 3, graphics etching; Step 4, clean, spare after passing test.

\section{RESULT AND ANALYSES}

\section{A. Key technique of washing, spin coating and soft baking}

After surface polished, dipping the slides for $15 \mathrm{~min}$ with the solution of $\mathrm{H}_{2} \mathrm{SO}_{4}: \mathrm{H}_{2} \mathrm{O}_{2}=3: 1$, then placing them in $120^{\circ} \mathrm{C}$ concentrated sulfuric acid, boiling about $10 \mathrm{~min}$. Then using ethanol, acetone and ionized water rinse them for $5 \mathrm{~min}$, drying the slides in the $150^{\circ} \mathrm{C}$ hot oven under a nitrogen atmosphere to enhance the activity of the glass surface about 3 minutes.

We used KW-4A desktop spin coating machine to spin coating the thick photoresist on the slide. During this, we need to pay attention to the following aspects:

- Note the introduction of air bubbles, pay attention to the timely removal the bubble of the plastic;

- The slide should be as far as possible be placed in the center of loading platform sucker, to prevent the spin coating process to shake;

- Select the appropriate speed, the slow buffer stage should be set up faster and shorter especially;

The time of spin coating is $60 \mathrm{~s}$, speed is $1000 \sim 5000 \mathrm{rad} / \mathrm{min}$. Under test control variable method, we test the relationship between speed and the photoresist thickness, and the relationship between speed and the tolerance time, as shown in Fig. 1.

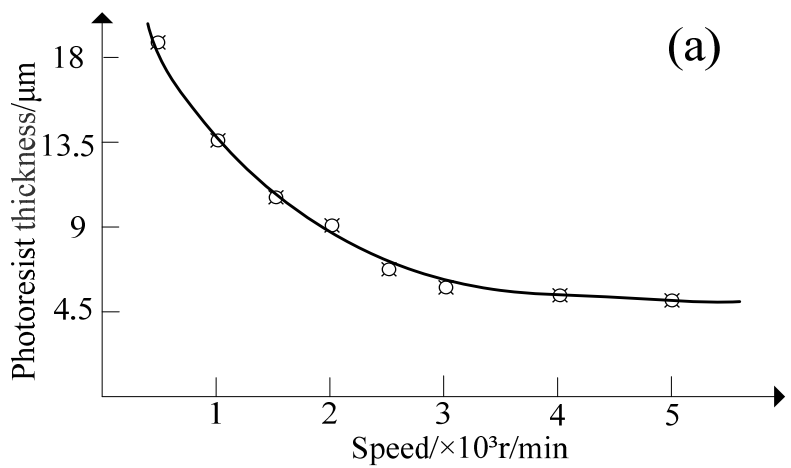

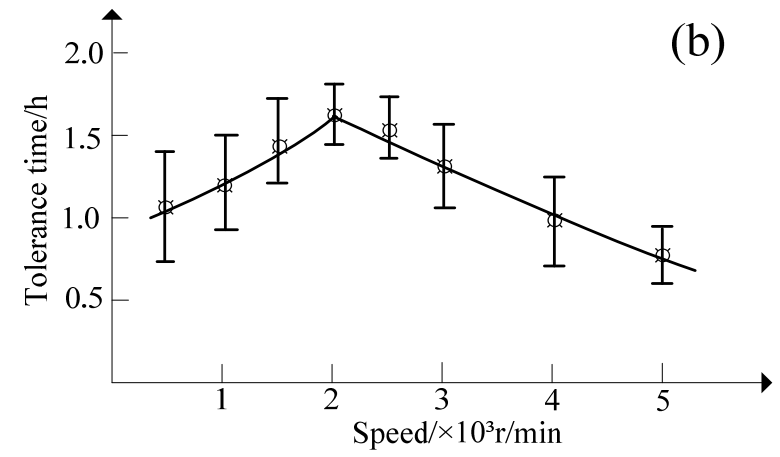

Figure 1. Speed and the thickness (a) speed and the tolerance time (b).

It is found that the photoresist thickness decreases with the speed to accelerate, after speed up to $3000 \mathrm{rad} / \mathrm{min}$, the photoresist thickness is no longer significantly reduced; under the same experimental conditions, the tolerance time of the photoresist in the etching solution with the speed increases to first increase and then reduction, the maximum tolerance time is at $2000 \mathrm{rad} / \mathrm{min}$. Because of the slow spin coating, the photoresist layer is thick, the adhesion of the glass matrix and photoresist layer is not strong; when the speed is too fast, the photoresist layer is too thin, and it may decrease the resistance to erosion. At the speed of $2000 \mathrm{rad} / \mathrm{min}$, the thickness is $9 \sim 10 \mu \mathrm{m}$, it would get the best quality, good surface uniformity and the longest tolerance time in the etching solution. The photoresist layer surface roughness curve is shown in Fig.2 (measured by AlphaStep P-6 stephight profiler), it beats about $\pm 40 \AA$. High-speed rotation of the slide causes more obvious standing wave phenomenon at the vertical direction. Photoresist thickness greater than $5 \mu \mathrm{m}$, standing wave effect can be no need to consider [8].

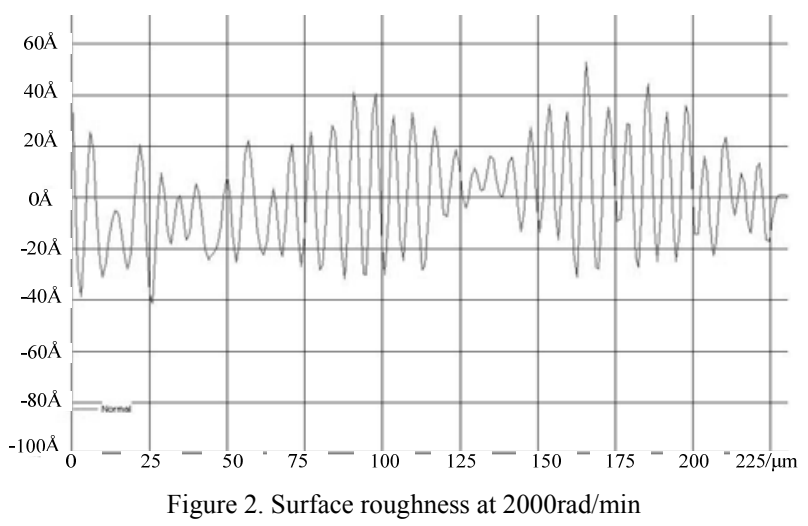

\section{B. Key technique of exposing, PEB and developing}

In theory, according to that wet etching is an isotropic etching, the edge of the mask graphic should be indented the same size of the depth of the design size characteristics. However, the actual etching process is rarely perfect isotropic, many tests proved that to etch 
$80 \mu \mathrm{m}$ depth, the edge of the mask graphic should be indented approximately $20 \mu \mathrm{m}$ under the conditions of this article.

The thick photoresist expose needs high UV light intensity. The URE-2000B deep ultraviolet lithography machine which was developed by the Chinese Academy of Sciences Institute of Optoelectronic Technology has a UV intensity of $15.7 \mathrm{~mW} / \mathrm{cm}^{2}$. It did well in the contact exposure. After exposure, in order to speed up the imaging speed, the post exposure baking (PEB) is needed. The test proved the bake time should not exceed 30 s, temperature should be controlled between $70 \sim 80^{\circ} \mathrm{C}$.

Trial and error for exposure of UV light of $9 \sim 10 \mu \mathrm{m}$ AZ P4620 photoresist on glass slides shown that: excessive exposure will cause the surface chemical reaction, degeneration and blistering, impact the edge quality of graphics as shown in Fig. 3(a); too little exposure will cause that the UV light can not throughout the layer, bottom film be left. The diffraction of a coherent state field will cause the PAC concentration uneven distribution after photochemical reaction, the graphical bottom won't be flat $[9,10]$, as shown in Fig. 3(b).

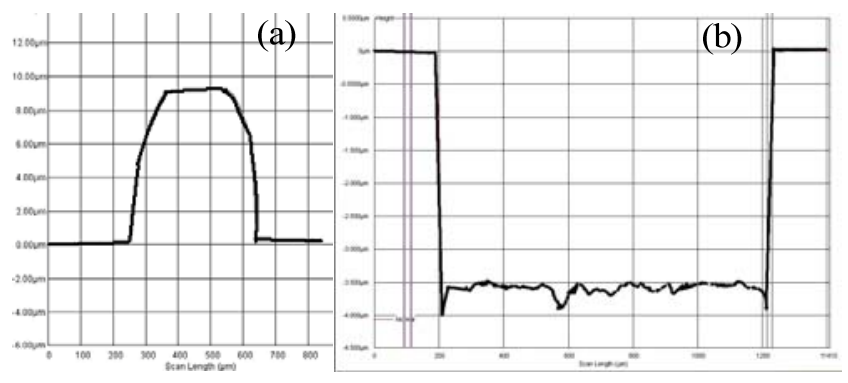

Figure 3. Overexposure (a) and underexposure (b)

According to the process that Table 1 shows, the test results showed that: controlling the exposure time at $225 \sim 250 \mathrm{~s}$, development time at $2 \sim 2.5 \mathrm{~min}$, it's able to obtain more satisfactory results. Not only the UV completely throughout the photoresist layer, but also to meet the requirements of steep graphics edge. The tolerance time could meet the requirements.

According to the summary of test results, compared with ordinary silicon wafer thin photoresist lithography process, the process this paper described has the following characteristics: increasing the exposure within the maximum allowable exposure, while reducing the developing time as far as possible.

TABLE 1. EXPOSING AND DEVELOPING PROCESS PARAMETERS

\begin{tabular}{|c|c|c|c|}
\hline Exposure time/s & Development time/s & Graphics quality & Tolerance time/min \\
\hline 150 & 480 & Bottom film & About 85 \\
\hline 200 & 300 & Thin film & 90 \\
\hline 225 & 180 & Good & 95 \\
\hline 250 & 150 & Good & 90 \\
\hline 275 & 120 & Edge isn't steep & About 90 \\
\hline 300 & 120 & Mild edge slope & About 85 \\
\hline
\end{tabular}

IV. EXPERIMENTAL RESULT

Combined with the results, the optimized process steps and parameters are shown in Table 2.

TABLE 2 THE OPTIMIZED PROCESS STEPS AND PARAMETERS

\begin{tabular}{|c|l|}
\hline Process & \multicolumn{1}{|c|}{ Parameters and methods } \\
\hline Cleaning & Concentrated sulfuric acid boiling; ethanol washing; de-ionized water rinsing \\
\hline Drying & $150^{\circ} \mathrm{C}, 5 \mathrm{~min}$; cooling under a nitrogen atmosphere \\
\hline Spin coating & $\mathrm{AZ} 4620,2000 \mathrm{rad} / \mathrm{min}, 1 \mathrm{~min}$ \\
\hline Soft bake & $80^{\circ} \mathrm{C}, 40 \sim 60 \mathrm{~min}$ \\
\hline Exposure & $15.7 \mathrm{~mW} / \mathrm{cm}^{2}, 225 \sim 250 \mathrm{~s}$ \\
\hline PEB & $80^{\circ} \mathrm{C}, \leqslant 30 \mathrm{~s}$ \\
\hline Developer & $\mathrm{AZ} 400 \mathrm{~K}(1: 3), 2 \sim 2.5 \mathrm{~min}$ \\
\hline Hard baking & $80 \sim 90^{\circ} \mathrm{C}, 70 \sim 90 \mathrm{~min}$ \\
\hline Etching & $\mathrm{BOE}(1: 10$ or $1: 15), 20 \sim 30^{\circ} \mathrm{C}$, Stirring, $800 \mathrm{rad} / \mathrm{min}, \leqslant 90 \mathrm{~min}$ \\
\hline Ccleaning & Followed cleaning by acetone, ethanol;de-ionized water rinsing; drying \\
\hline
\end{tabular}


According to the actual testing process parameters it's able to achieve a maximum tolerance time of $90 \sim 110 \mathrm{~min}$ and etching depth of about $80 \mu \mathrm{m}$.

\section{CONCLUSIONS}

This article uses thick photoresist wet etching process in soda-lime slide glass substrate prepare pipeline for microfluidic chips, and gets the depth of $80 \mu \mathrm{m}$, the feature size of $50 \mu \mathrm{m}$, the sidewall steepness of less than $100^{\circ}$, the bottom flatness error of less than $\pm 1.5 \mu \mathrm{m}$, the production cycle of about 4 hour. We finished a comprehensive and systematic study, such as the square glass slide cleaning process, the photoresist thickness control, the temperature and time of various stages heat drying, exposing and developing process parameters, the dilution and composition ratio of BOE. The process is simple, fast, and low cost, it has a good prospect of mass production. It has been applied to the production of a certain microfluidic detection chips.

\section{REFERENCES}

[1] Zhao Y, Gong Y, Zhang W 2011 Acta Optica Sinica 3111

[2] Richardson D J, Nilsson J, Clarkson W A 2010 J. Opt. Soc. Am. B 2711

[3] Cheng C W, Shen W C, Lin C Y, et al. 2010 Appl. Phys. A. Mater. 1012

[4] Dentinger P M, Krafcik K L, et al. 2002 Microelectronic Engineering 6162

[5] Huang W D, Zhang H, Xu T, et al. 2011 Chinese Sci Bull (Chinese Ver) 56

[6] Hirata Y 2007 Nuclear Instruments and Methods in Physics Research B 208 1-4

[7] Wang H, Huang H Q, Dai Z P, et al. 2005 Chem. J. C. U. 2611

[8] Xiao X, Yang J, Du J L, et al. 2002 SPIE 4924

[9] Franssila S 2005 Introduction to microfabrication (Helsinki: Wiley Press)

[10] Roulet J C, Volkel R,. Herzig H P, et al. 2001 Journal of Microelectromechanical Systems 10 